\title{
Research Progress on Iridoid Glycosides and Pharmacological Effects of Zuogui Wan
}

\author{
Kaixia Xu, Yafei Guo, Yingli Wang and Qianjin Feng* \\ Shanxi University of Chinese Medicine, China \\ *Corresponding author: Qianjin Feng, Shanxi University of Chinese Medicine, Jinzhong 030619, China
}

\begin{tabular}{l} 
ARTICLE INFO \\
Received: 蔧 February 20, 2020 \\
Published: 㓞 February 28, 2020 \\
\hline
\end{tabular}

Citation: Kaixia X, Yafei G, Yingli W and Qianjin F. Research Progress on Iridoid Glycosides and Pharmacological Effects of Zuogui Wan. Biomed J Sci \& Tech Res 26(1)2020. BJSTR. MS.ID.004304.

\section{ABSTRACT}

Zuogui Wan (ZGW) has been used as a typical recipe for tonifying kidney essence in Traditional Chinese Medicine (TCM). The iridoid glycosides active ingredient are the important material basis for efficacy.

Keywords: Zuogui Wan; Iridoid Glycosides; Pharmacological Effect

Abbreviations: ZGW: Zuogui Wan; TCM: Traditional Chinese Medicine; ZGWRS: ZGW Contained Rat Serum; BGP: Bone Gla-Protein; IOP: Intraocular Pressure; LDP: Density Lipoprotein

\section{Mini Review}

The formula of Zuogui Wan (ZGW) is from a traditional Chinese medicine (TCM) book titled "Jingyue Quanshu". It has been used as a classic recipe for tonifying TCM kidney, such as diabetic nephropathy, for hundreds of years. ZGW consists of Rehmannia glutinosa (Shu Di Huang), Cuscuta chinensis (Tu Si Zi), Cornus officinalis (Shan Zhu Yu), Lycium barbarum (Gou Qi Zi), Dioscorea opposite (Shan Yao), and Cyathula officinalis (Chuan Niu Xi), etc. As a valuable TCM, ZGW has also been used to treat osteoporosis and type 2 diabetic nephropathy. Ju et al. reported that ZGW could prevent and treat osteoporosis [1] In addition, ZGW can significantly increase Gla-containing protein content and reduce calcitonin content in osteoporosis rats without ovaries. The composition of TCM is remarkably complicated, especially after entering in the body. The therapeutic effect of TCM is attributed to the synergistic effects of its multiple components. Serum pharmacochemistry has been introduced to the study on therapeutic material basis of TCM $[2,3]$.

The results indicate that the active components in the ZGW contained rat serum (ZGWRS) prepared by administering rats with ZGW can significantly influence the forming of osteocalcin and the secretion of bone Gla-protein (BGP) by Gegenbaur cells in vitro and improve hyperplasia and the differentiation of Gegenbaur cells most likely by estrogenic effects to adjust ERK/Smads signal channels [4-6]. In addition, ZGWRS can inhibit the apoptosis of thymocytes induced by corticosterone, possibly by regulating the ratio of Bcl2 to Bax [7]. ZGWRS with complicated chemical composition and multiple-target features can exert a systematic influence on rats.

\section{Chemical Compositions and Pharmacological Effects}

Morroniside, loganin, sweroside, loganic acid and 8-epiloganic acid are the bioactive herbal ingredients isolated from Cornus officinalis (Shan Zhu Yu) that is a major herb component of ZGW. Morroniside and loganin are the compounds from Cornus officinalis (Shan Zhu Yu). It has been reported that morroniside and loganin can improve the morphological changes of rat mesangial cells and regulate their growth by reducing oxidative stress, which provides a molecular mechanism for the use of morroniside and loganin in the early stages of diabetic nephropathy [8]. In addition, loganin can significantly inhibit the expression of fibronectin and IL-6 that are harmful to the mesangial cells in kidney [9]. Sweroside can attenuate and inhibit apoptosis and has a direct osteogenic effect on the proliferation and differentiation of human MG-63 cells and rat osteoblasts in vitro [10]. Cornus officinalis (Shan Zhu Yu) has been safely used for the treatment of osteoporosis in postmenopausal women or elderly men in Asia with a long history.Sweroside is one of the main bioactive herbal ingredients in ZGW. 
Loganic acid is an active iridoid in Cornus officinalis (Shan $\mathrm{Zhu} \mathrm{Yu}$ ) and is a major polar compound of ZGW. A single dose of $0.7 \%$ loganic acid extract in vehiculum containing $0.15 \%$ sodium hyaluronate administered directly into the conjunctival sac of animal model (New Zealand rabbits) can decrease $15 \%$ intraocular pressure (IOP) in $60 \mathrm{~min}$ in average. Loganic acid itself can decrease IOP $\sim 23 \%$, indicating its potential application in ocular hypertension therapy [11]. Coumaric acid, a hydroxyl derivative of cinnamic acid, is a bioactive herbal ingredient from Lycium barbarum (Gou Qi Zi). It can reduce the peroxidation of low density lipoprotein (LDL) and has shown biological functions including anti-mutagenesis, anti-genotoxicity, anti-microbial, and antioxidant activities. Coumaric acid can also inhibit cellular melanogenesis and plays important roles in immune regulation. In addition, coumaric acid can capture peroxide substance and reduce the incidence of vascular atherosclerosis. [12]. Kaempferol3-glucuronide is a bioactive herbal ingredient from Cuscuta chinensis (Tu Si Zi). Kaempferols can be efficiently absorbed by human body, even at low oral doses. Kaempferol-3-glucuronide is its major metabolite found in plasma and urine [13]. Studies have shown that dietary kaempferol can reduce the risk of chronic diseases, especially cancers. Kaempferol may augment antioxidant in body to against free radicals, preventing the development of cancer [14]. 5-Hydroxymethyl-2-furfural glucuronide and dihydro5-hydroxymethyl-2-furfural glucuronide are bioactive herbal ingredients from Rehmannia glutinosa (Shu Di Huang). They can be hydrolyzed to 5-hydroxymethyl-2-furfural in vivo and have almost same functions. 5-(hydroxymethyl)-2-furfural (5-HMF) has many biological functions, such as antioxidant activity, inhibiting sickling of red blood cells, and hypoxic injury $[15,16]$.

\section{Funding}

Shanxi Province Key Research and Development Program for the International Cooperation (201703D421031).

\section{Competing interests}

The authors declare that they have no competing interests.

\section{References}

1 Ju D, Wu P, Jia H, Yu Z (2003) Effect of Zuogui pill on the content of bone gla -containing Protein and calcitonim in ovariectomy-induced osteoporosis rats. Chin J Info Tradit Chin Med 10(1): 16-17.

\section{ISSN: 2574-1241}

DOI: $10.26717 /$ BJSTR.2020.26.004304

Qianjin Feng. Biomed J Sci \& Tech Res

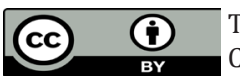

This work is licensed under Creative Commons Attribution 4.0 License

Submission Link: https://biomedres.us/submit-manuscript.php
2 Zhang JH, Xing J, Fan LX (2017) Intervention effects of Zuoguiwan containing serum on osteoblast through ERK1 /2 and Wnt / $\beta$-catenin signaling pathway in models with kidney Yang-deficiency, kidney-Yindeficiency osteoporosis syndromes. China J Chin Mater Med 42(20): 3983-3989.

3 Chen DY, Lin SR, Deng YY (2018) Expressions of GPR48 MMP6 and ATF4 of Bone Marrow Mesenchymal Stem Cells by Serum Containing Zuogui Pills. Liaoning J Tradit Chin Med 45(2): 415-417.

4 Liu SQ Yang Z, Liu LP (2018) Discussion on Prevention and Treatment Using Zuogui Pill in Imbalance of Intestinal Homeostasis Caused by Senility Based on Eryang Weiwei. J Tradit Chin Med 59(16): 1380-1382.

5 Meng Y, Ren YL, Sun YJ (2016) Impact of Zuogui Pill,Yougui Pill and Their Decomposed Recipes on the Expression of Renal Alkaline Phosphatase and Osteocalcin in Ovariectomized Osteoporosis Model Rats [J]. J Tradit Chin Med 57(5): 423-427.

6 Liu LP, Bai LF, Jiang B (2017) Effects of Bushen Tianjing Recipe containing serum on osteoblast functions via ER/ERK pathway. Pharmacol Clin Chin Mater Med 33(2): 6-9.

7 Hao C, Ren Y, Zhao J (2012) Effects of Zuogui pill medicated serum via ERK/Samds dependent pathway on MC3T3-E1 cell gene expression. Chin Pharmacol Bull 28(6): 872-876.

8 Xu H, Shen J, Liu H, Shi Y, Li L, et al. (2006) Morroniside and loganin extracted from Cornus officinalis have protective effects on rat mesangial cell proliferation exposed to advanced glycation end products by preventing oxidative stress, Can J Physiol Pharm 84(12): 267-1273.

9 Ma W, Wang K, Cheng C, Yan G, Lu W, et al. (2014) Bioactive compounds from Cornus officinalis fruits and their effects on diabetic nephropathy. J Ethnopharmacol 153(3): 840-845.

10 Sun H, Li L, Zhang A, Zhang N, Lv H, et al. (2013) Protective effects of sweroside on human MG-63 cells and rat osteoblasts. Fitoterapia (84): 174-179.

11 Szumny D, Kucharska AZ, Piorecki N, Szumny A, Ozanski T, et al. (2014) Ocular hypotensive properties of Cornus mas extract and loganic acid. Acta Ophthalmo.

12 Kilic I, Yesiloglu Y (2013) Spectroscopic studies on the antioxidant activity of p-coumaric acid. Spectrochim Acta, A 115: 719-724.

13 Du Pont MS, Day AJ, Bennett RN, Mellon FM, Kroon PA (2004) Absorption of kaempferol from endive, a source of kaempferol-3-glucuronide, in humans. Eur J Clin Nutr 58(6): 947-954.

14 Chen A, Chen Y (2013) A review of the dietary flavonoid, kaempferol on human health and cancer chemoprevention,.Food Chem 138(4): pp. 2099-2107.

15 Li M, Wu L, Zhao T, Xiong L, Huang X, et al. (2011) The protective role of 5-HMF against hypoxic injury, Cell Stress Chaperones 16(3): 267-273.

16 Zhang J, Di Y, Wu L, He Y, Zhao T, et al. (2015) 5-HMF prevents against oxidative injury via APE/Ref-1.Free Radic Res 49(1): 86-94.

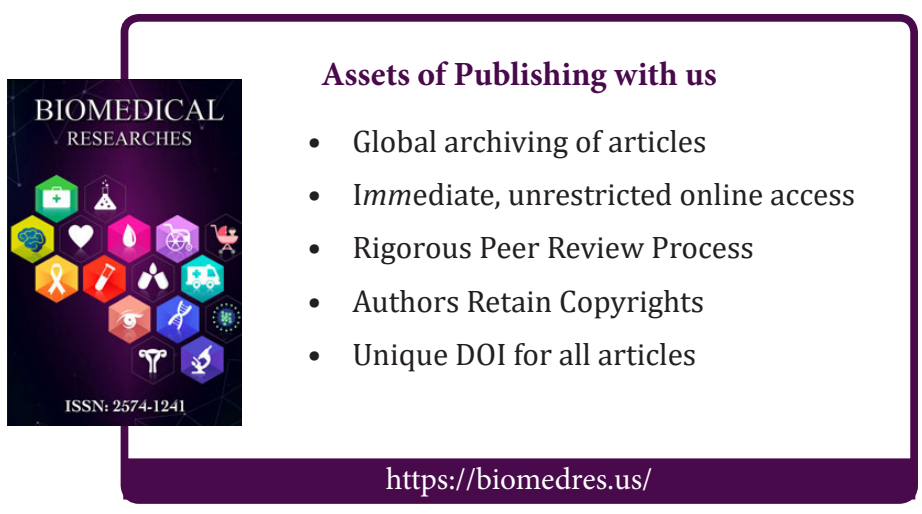

Copyright@ Qianjin Feng | Biomed J Sci \& Tech Res | BJSTR. MS.ID.004304. 